\title{
POSIÇÃO DOS FRUTOS E SEU EFEITO NA REPARTIÇÃO DA MATÉRIA SECA DA PLANTA DO TOMATEIRO
}

\author{
FRUIT POSITION AND ITS EFFECTS ON DRY MATTER DISTRIBUTION OF THE TOMATO PLANT
}

\author{
Jerônimo Luiz Andriolo ${ }^{1}$ Loeni Ludke ${ }^{2}$ Tatiana da Silva Duarte $^{3}$ \\ Etiana Caldeira Skrebsky ${ }^{2}$
}

RESUMO

Plantas de tomateiro foram cultivadas no interior de estufas de polietileno no decorrer da primavera e do outono com o objetivo de determinar o efeito da posição dos frutos sobre a distribuição da matéria seca entre as partes vegetativas e os frutos. Os tratamentos foram constituídos por plantas conduzidas com uma haste (controle) ou duas hastes por planta, com frutos localizados somente sobre a haste principal ou distribuídos sobre as duas hastes. Em cada época, o número de inflorescências por planta foi mantido idêntico em todos os tratamentos, igual a oito na primavera e dez no outono, enquanto o número de folhas por planta foi, respectivamente, 27 e 37 nas plantas-controle e 52 e 65, em média, nas plantas com duas hastes. Na primavera, as plantas com duas hastes acumularam uma média de $155 \mathrm{~g}$ de matéria seca de frutos, e aquelas com uma haste apenas $95 \mathrm{~g}$. No outono, esse parâmetro não mostrou diferença significativa entre os tratamentos: valores de $99 \mathrm{~g}$ nas plantas com uma haste e uma média de 78 g naquelas com duas hastes. Nos dois experimentos, não foram observadas diferenças significativas entre os tratamentos com duas hastes por planta, independentemente da posição dos frutos sobre a planta. No outono, a fração da matéria seca total alocada para os frutos foi de 0,40 nas plantas com uma haste e em média de 0,24 nas plantas com duas hastes, indicando uma possível redução na força de dreno dos frutos. Concluiu-se que a posição dos frutos não modificou a distribuição da matéria seca da planta e que os resultados suportaram a hipótese de um "pool" único de assimilados circulando livremente no interior da planta do tomateiro.

Palavras-chave: Lycopersicon esculentum, crescimento, matéria seca, repartição de assimilados.

\section{SUMMARY}

Tomato plants were grown in Spring and Fall in a non-heated greenhouse in order to investigate the effect of fruit position on dry matter distribution between vegetative organs and fruits. Treatments consisted of plants with one shoot (control) or two shoots per plant, with fruits located in both stems or only in the main stem. In each experiment, the same number of trusses was kept in all plants, by truss removal on the double-shoot plants. Thus, truss number per plant was eight in Spring and ten in Fall, while number of leaves per plant was respectively 27 and 37 in control plants and 52 and 65, on average, on the doubleshoot plants. In Spring, mean fruit dry matter was $155 \mathrm{~g}$ on double-shoot plants and $95 \mathrm{~g}$ on control plants. In Fall, mean fruit dry matter accumulation was $99 \mathrm{~g}$ in double-shoot and $78 \mathrm{~g}$ in single-shoot plants and differences were not significant. In both seasons, no significant differences were recorded between double-shoot treatments, regardless of the position of fruits on the stem. In Fall, the fraction of total dry matter allocated to fruits was 0.40 in control and 0.24, on average, on the double-shoot plants, suggesting sink strength of fruits was affected by low temperatures. It was concluded that fruit position do not affect dry matter distribution and results support the hypothesis of one common pool of assimilates circulating freely in the tomato plant.

Key words: Lycopersicon esculentum, growth, dry matter, partitioning of assimilates.

\section{INTRODUÇÃO}

O esquema de distribuição dos assimilados entre os órgãos da planta do tomateiro, que é atualmente utilizado pelos fisiologistas, considera os assimilados sendo produzidos pelos órgãos fontes e exportados para os órgãos drenos. A força de dreno é definida como sendo a habilidade que tem um determinado dreno para atrair assimilados para seu

\footnotetext{
${ }^{1}$ Engenheiro Agrônomo, Professor Titular, Doutor, Departamento de Fitotecnia, Universidade Federal de Santa Maria (UFSM). 97105900, Santa Maria, RS. E-mail: andriolo@ creta.ccr.ufsm.br. Autor para correspondência.

${ }^{2}$ Acadêmico do Curso de Agronomia da UFSM, Bolsista do CNPq-PIBIC-UFSM.

${ }^{3}$ Acadêmico do Curso de Agronomia da UFSM. 
próprio crescimento. Uma planta é descrita como um conjunto de órgãos drenos, regidos por relações competitivas entre as fontes e os drenos e também entre os diferentes drenos da planta (WARREN-WILSON, 1972; DE KONNING, 1994). HO (1988) definiu a força de dreno potencial de um fruto de tomateiro como sendo o crescimento máximo atingido por esse fruto quando nenhuma limitação existe em nível do suprimento de assimilados. Os conhecimentos atualmente existentes no campo da fisiologia da produção ainda não permitem afirmar se a planta de tomateiro se subdivide em vários conjuntos de unidades fontes-drenos ou se existe um "pool" único de assimilados que circula livremente no interior da planta e supre todos os drenos segundo suas necessidades.

Evidências favoráveis à primeira hipótese provêm dos trabalhos de BONNEMAIN (1968) e de HO \& HEWITT (1986). Esses autores observaram frutos de tomateiro sendo supridos preferencialmente pelas folhas adjacentes, e seus resultados serviram de suporte para a descrição dessa planta como sendo formada pelo conjunto de sucessivas unidades compostas por três folhas e uma inflorescência.

A hipótese de um "pool" único de assimilados foi utilizada por vários pesquisadores no desenvolvimento de modelos mecanísticos para simular a repartição da matéria seca e o rendimento de frutos do tomateiro (JONES et al., 1991; HEUVELINK \& MARCELIS, 1989; DE KONNING, 1994). Entretanto, dados conclusivos sobre a questão ainda são escassos e essa última hipótese foi utilizada principalmente para simplificar a estrutura dos modelos (CHALLA, 1985). Em um artigo recente, HEUVELINK (1995) mostrou que a distância entre as fontes e os drenos tem pequena importância na planta do tomateiro, reforçando a hipótese do "pool" único de assimilados. Entretanto, seus dados foram obtidos em estufas aquecidas artificialmente, com pequenas flutuações diárias na temperatura do ar, dentro dos limites de $24^{\circ} \mathrm{C}$ durante o dia e de $18^{\circ} \mathrm{C}$ durante a noite. Em outro trabalho, MARCELIS \& DE KONNING (1995) mostraram que a temperatura é o mais importante elemento meteorológico que afeta a distribuição da matéria seca da planta do tomateiro. Dessa forma, existem dúvidas quanto à validade da conclusão de HEUVELINK (1995) em condições ambientais não controladas, em que a amplitude térmica diária é elevada.

O presente trabalho investigou o efeito da posição dos frutos na distribuição da matéria seca de plantas de tomateiro, cultivadas no interior de estufas não aquecidas, localizadas na região Sul do Brasil, onde a temperatura do ar pode atingir valores superiores a $35^{\circ} \mathrm{C}$ durante o dia e cair para valores inferiores a $10^{\circ} \mathrm{C}$ durante a noite, a fim de testar a validade da conclusão de HEUVELINK (1995) nessas condições.

\section{MATERIAL E MÉTODOS}

Os experimentos foram conduzidos no Departamento de Fitotecnia da Universidade Federal de Santa Maria, RS, (latitude: 29\%43'S, longitude: 534' $\mathrm{W}$, altitude: $95 \mathrm{~m}$ ). As mudas de tomateiro (Lycopersicon esculentum Mill.), híbrido longavida "Diva", foram produzidas em bandejas de poliestireno e, no estádio de 6 folhas definitivas, foram transplantadas para o solo no interior de uma estufa de polietileno transparente $(10 \mathrm{~m}$ de largura, $50 \mathrm{~m}$ de comprimento e 4,5m de altura). O manejo do ambiente da estufa foi efetuado apenas com ventilação natural, através da abertura das cortinas laterais às 9 horas e fechamento às 15 horas, aproximadamente.

A densidade utilizada foi de 2,2 plantas $\mathrm{m}^{-2}$, em fileiras simples $(1,0 \mathrm{~m}$ entre fileiras e $0,45 \mathrm{~m}$ entre plantas). As plantas foram conduzidas verticalmente através de fitas plásticas, removendo-se as ramificaçõs axilares uma vez por semana. Um hormônio vegetal (citocinina na concentração $8 \mathrm{x}$ $10^{-5} \%$ ) foi aplicado duas vezes por semana durante o período de inverno, quando as temperaturas diurnas se situaram em valores inferiores a $18^{\circ} \mathrm{C}$, a fim de estimular o crescimento dos frutos jovens e compensar possíveis falhas na polinização (FAO, 1990).

A adubação de manutenção foi efetuada com base no resultado da análise química do solo e de acordo com as recomendações de VEDUIN \& BARTZ (1998) para a cultura do tomateiro em estufas. A irrigação foi realizada de forma a manter a tensão da água no solo em valores inferiores a $-0,5 \mathrm{MPa}$, medida por dois tensiômetros instalados aleatoriamente sobre as fileiras e entre as plantas, a $15 \mathrm{~cm}$ de profundidade. A tabela 1 resume as informações relativas aos experimentos realizados.

Os experimentos constaram de plantas conduzidas com uma e duas hastes por planta. Para obter plantas com duas hastes, a primeira brotação axilar imediatamente abaixo da primeira inflorescência foi mantida e conduzida, verticalmente, da mesma maneira que a haste principal. Dessa forma, duas hastes de altura similar existiam na mesma planta. A densidade de plantas foi ajustada de forma a manter constante a densidade de hastes por uni- 
Tabela 1 - Datas de semeadura, transplante e conclusão, duração (dias após a semeadura) dos experimentos, radiação solar global no exterior da estufa e médias das temperaturas máxima, mínima e média diária do ar no interior da estufa calculadas no período entre o transplante e a conclusão dos experimentos. Santa Maria, RS, 1996 e 1997.

\begin{tabular}{|c|c|c|c|c|c|c|c|c|}
\hline \multirow[t]{2}{*}{ Experimento } & \multicolumn{3}{|c|}{ Datas } & \multirow[t]{2}{*}{$\begin{array}{l}\text { Duração } \\
\text { (Dias) }\end{array}$} & \multirow[t]{2}{*}{$\begin{array}{l}\text { Radiação solar } \\
\text { global }\left(\mathrm{MJ} \mathrm{m} \mathrm{m}^{2} \mathrm{~d}^{-1}\right)\end{array}$} & \multicolumn{3}{|c|}{$\begin{array}{c}\text { Médias das temperaturas } \\
\left({ }^{\circ} \mathrm{C}\right)\end{array}$} \\
\hline & Semeadura & Transplante & Final & & & Máxima & Mínima & Média \\
\hline Primavera & 08/08/96 & 03/09/96 & $26 / 11 / 96$ & 115 & 14,9 & 32,4 & 15,6 & 24,3 \\
\hline Outono & $20 / 02 / 97$ & $19 / 03 / 97$ & $11 / 07 / 97$ & 111 & 12,8 & 27,2 & 11,3 & 19,3 \\
\hline
\end{tabular}

e pedúnculos) e dos frutos foi determinada após secagem em estufa a $60^{\circ} \mathrm{C}$, durante uma semana. O número de folhas $(>0,5 \mathrm{~cm})$, inflorescências visíveis e frutos (diâmetro $>0,5$ $\mathrm{cm})$ também foram registrados. Os resultados obtidos no final dos experimentos foram submetidos à

dade de superfície de solo. Os tratamentos constaram de: i) plantas conduzidas com apenas uma haste (testemunha) e com todas as inflorescências distribuídas normalmente sobre a haste principal, da mesma forma como é praticado na produção comercial; ii) plantas com duas hastes e com o mesmo número de inflorescências que as plantas testemunhas, porém distribuídas alternadamente na haste principal e na secundária (S2F2), através da remoção sistemática ainda na fase de botão das inflorescências excedentes; iii) plantas com duas hastes e com o mesmo número de inflorescências que as plantas testemunhas, porém localizadas apenas na haste principal (S2F1), através da remoção sistemática de todas as inflorescências emitidas na haste secundária (Figura 1). O delineamento experimental utilizado foi de blocos casualizados, com quatro repetições e 30 plantas por parcela.

As temperaturas máximas e mínimas diárias do ar no interior da estufa foram registradas diariamente com o auxílio de termômetros. A média dessas duas temperaturas forneceu a temperatura média diária do ar. A radiação solar global no exterior da estufa foi estimada a partir do brilho solar diário, através do modelo de Angstron, com os coeficientes ajustados para Santa Maria por ESTEFANEL $\boldsymbol{e}$ t al. (1990). O brilho solar diário foi obtido na Estação Climatológica do Departamento de Fitotecnia, localizada a, aproximadamente, $150 \mathrm{~m}$ da área experimental. A transmissividade média da estufa foi considerada como igual a 0,73 (BURIOL et al., 1995).

A cada sete dias na primavera e quatorze dias no outono, duas plantas de cada tratamento foram coletadas ao acaso dentro das repetições para efetuar medidas destrutivas do crescimento e do desenvolvimento. Plantas localizadas nas bordaduras das coletas precedentes não foram utilizadas. A matéria seca das folhas, do caule (incluindo pecíolos análise da variância e a significância das diferenças entre as médias foi testada pelo teste $t$.

\section{RESULTADOS E DISCUSSÃO}

\section{Desenvolvimento das plantas}

O número de inflorescências por planta foi mantido idêntico em todos os tratamentos, totalizando oito no final do experimento de primavera e dez no outono. O número de folhas na última coleta das plantas conduzidas com uma haste (controle) totalizou 27 na primavera e 37 no outono, correspondendo a, aproximadamente, 3,4 e 4,6 folhas por inflorescência (Tabela 2 e Figura 2a,b). No mesmo período, comparando-se somente as médias das plantas com duas hastes (S2F2 e S2F3) cultivadas

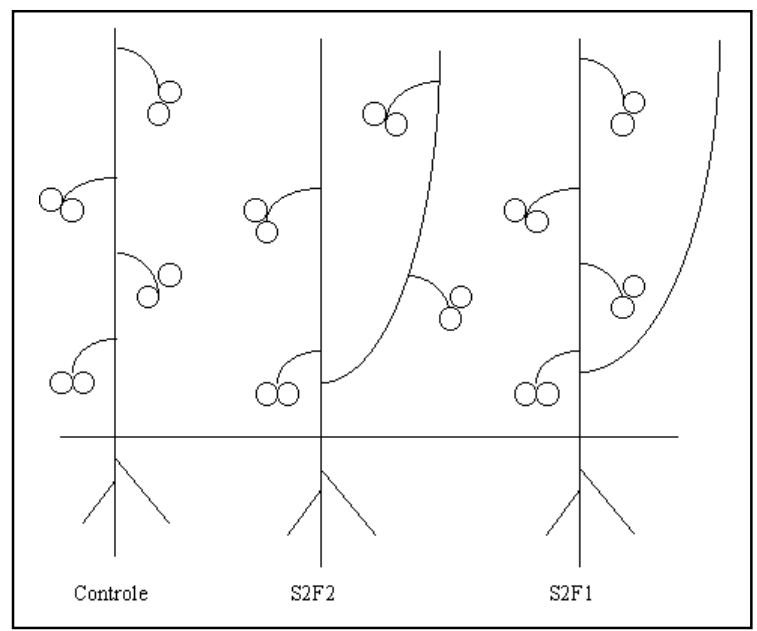

Figura 1 - Representação esquemática dos tratamentos. As plantas controle foram conduzidas com uma haste por planta; as plantas S2F2 com duas hastes e inflorescências distribuídas sobre as duas hastes e as plantas S2F1 com duas hastes e inflorescências localizadas somente na haste principal. Santa Maria, RS, 1996 e 1997. 
Tabela 2 - Valores médios por planta do número de folhas e de frutos, da matéria seca (MS) total e de frutos (g) e da fração da matéria seca alocada para os frutos (FDMF) no final dos experimentos. Santa Maria, RS, 1996 e 1997.

Tratamentos $\mathrm{N}^{\circ}$ de folhas $\mathrm{N}^{\circ}$ de frutos MS total MS frutos FDMF

\begin{tabular}{|c|c|c|c|c|c|}
\hline \multicolumn{6}{|l|}{ Primavera } \\
\hline Controle & $27 a^{1}$ & $33 \mathrm{a}$ & $258 \mathrm{a}$ & $95 \mathrm{a}$ & $0,37 \mathrm{a}$ \\
\hline $\mathrm{S} 2 \mathrm{~F} 2$ & $52 \mathrm{~b}$ & $32 \mathrm{a}$ & $396 \mathrm{~b}$ & $140 \mathrm{~b}$ & $0,35 \mathrm{a}$ \\
\hline $\mathrm{S} 2 \mathrm{~F} 1$ & $53 \mathrm{~b}$ & $34 \mathrm{a}$ & $400 \mathrm{~b}$ & $171 \mathrm{~b}$ & $0,43 \mathrm{~b}$ \\
\hline CV (\%) & 4,0 & 6,6 & 10,9 & 15,4 & 5,4 \\
\hline \multicolumn{6}{|l|}{ Outono } \\
\hline Controle & 37 a & $24 \mathrm{a}$ & $248 \mathrm{a}$ & $99 \mathrm{a}$ & $0,40 \mathrm{a}$ \\
\hline $\mathrm{S} 2 \mathrm{~F} 2$ & $61 \mathrm{~b}$ & $22 \mathrm{a}$ & $307 \mathrm{~b}$ & $86 \mathrm{ab}$ & $0,28 \mathrm{~b}$ \\
\hline $\mathrm{S} 2 \mathrm{~F} 1$ & $68 \mathrm{c}$ & $22 \mathrm{a}$ & $346 \mathrm{~b}$ & $71 \mathrm{~b}$ & $0,20 \mathrm{~b}$ \\
\hline CV (\%) & 3,4 & 3,2 & 17,5 & 7,0 & 4,4 \\
\hline
\end{tabular}

${ }^{1}$ Médias seguidas pelas mesmas letras na posição vertical não diferem significativamente pelo teste $t$ a $\mathrm{p}=0,95$.

nas duas épocas, observaram-se valores de 52 folhas na primavera e 64 folhas por planta no outono, correspondendo a, aproximadamente, 6,5 e 8 folhas por inflorescência. A evolução do número de frutos por planta no transcorrer do período de realização dos dois experimentos não diferiu significativamente entre os tratamentos, porém valores mais baixos foram observados no outono (Figuras 2c, 2d). Dessa forma, em cada um dos experimentos, o tamanho do compartimento dreno das plantas foi similar em todos os tratamentos, enquanto o compartimento fonte foi maior nas plantas conduzidas com duas hastes.

\section{Acumulação de matéria seca}

Nos dois experimentos, as plantas com duas hastes acumularam mais matéria seca total do que aquelas com apenas uma haste. Porém, a diferença entre plantas com uma e com duas hastes começou a ser observada somente após os 80 dias na primavera, enquanto no outono essa diferença foi constatada mais precocemente aos 55 dias da semeadura, aproximadamente (Tabela 2 e Figuras 3a,b). A acumulação de matéria seca total não diferiu significativamente entre os tratamentos S2F2 e $\mathrm{S} 2 \mathrm{~F} 1$, nas duas épocas. A acumulação de matéria seca nos frutos no final do período foi maior na primavera e diferiu significativamente nas plantas com duas hastes, em relação àquelas com uma haste. Comportamento inverso foi observado no outono, quando as plantas $\mathrm{S} 2 \mathrm{~F} 1$ mostraram o menor valor desse parâmetro, diferindo significativamente das plantas com uma haste (Tabela 2 e Figura 3d).

A fração de matéria seca alocada para os frutos (FDMF) foi significativamente mais elevada na primavera para o tratamento S2F1, atingindo o valor de 0,43 (Tabela 2 e Figura 4a). No outono, essa fração foi significativamente menor nas plantas com duas hastes, atingindo o valor mais baixo no tratamento $\mathrm{S} 2 \mathrm{~F} 1$, igual a 0,20 (Tabela 2 e Figura 4b).

A força de dreno da planta do tomateiro tem sido associada com o número de frutos existentes sobre a planta (DE KONNING, 1994). Portanto, plantas com número semelhante de frutos deverão ter uma força de dreno similar. A hipótese da existência de vários conjuntos independentes de unidades fonte-dreno implica que as plantas com os frutos melhor distribuídos sobre as duas hastes deveriam ser favorecidas, apresentando uma maior acumulação de matéria seca de frutos. Essa hipótese não foi confirmada pelos experimentos realizados, uma vez que não foram verificadas diferenças significativas entre os tratamentos S2F2 e S2F1. Os dados sugerem, portanto, a existência do "pool" único de assimilados, confirmando as observações de HEUVELINK (1995, 1996) obtidas na Holanda e em condições controladas. Isso significa que os assimilados circulam livremente entre todos os drenos, independentemente de sua posição sobre

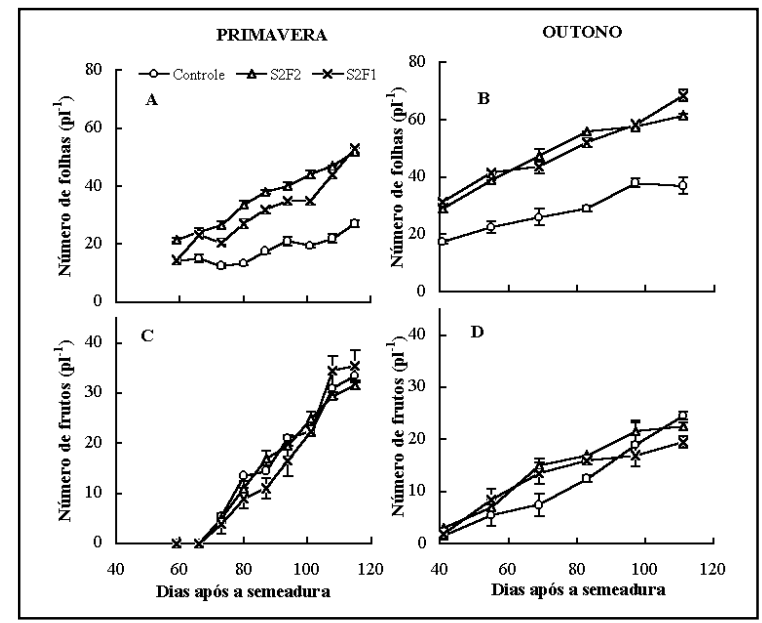

Figura 2 - Valores médios por planta do número de folhas e de frutos na primavera $(\mathrm{a}, \mathrm{c})$ e no outono $(\mathrm{b}, \mathrm{d})$. As barras verticais indicam o desvio-padrão da média de cada observação. Santa Maria, RS, 1996 e 1997. 


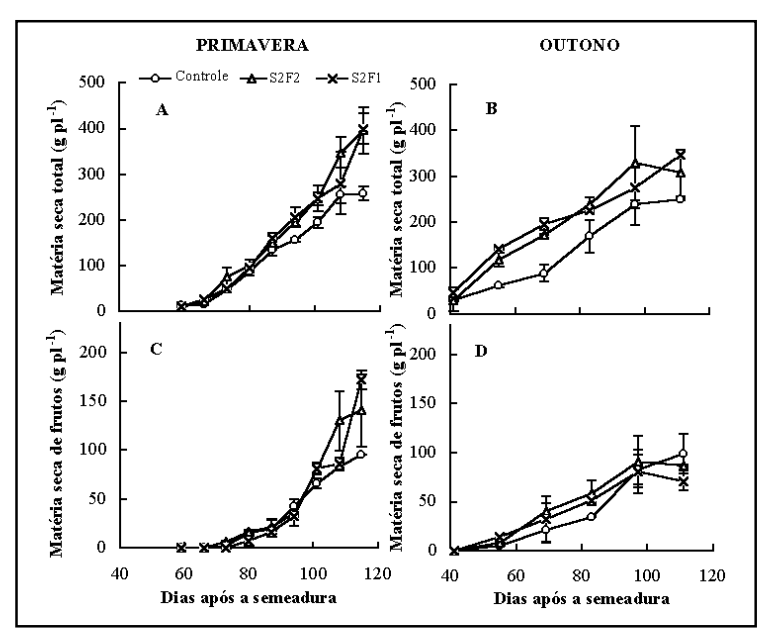

Figura 3 - Valores médios por planta da matéria seca total e de frutos na primavera e no outono $(b, d)$. As barras verticais indicam o desvio-padrão da média de cada observação. Santa Maria, RS, 1996 e 1997.

a planta, e o transporte não se constitui em fator limitante ao crescimento dos frutos. Quando os elementos meteorológicos, especialmente radiação solar e temperatura, mudaram da primavera para o outono, os tratamentos S2F2 e S2F1 induziram uma menor produção de matéria seca total. Entretanto, a produção de matéria seca de frutos nesses dois tratamentos não mostrou crescimento em relação às plantas utilizadas como controle. Ao contrário, o tratamento S2F1 induziu um valor de matéria seca significativamente menor que o controle (Tabela 2). MARCELIS \& BAAN-HOFMAN-EIJER (1993) sugeriram que a temperatura pode afetar a repartição da matéria seca da planta. Os resultados obtidos neste trabalho suportam a hipótese desses autores, porém indicam que a influência da temperatura não se exerce sobre a resistência ao transporte dos assimilados.

A carga de frutos aumenta a alocação de matéria seca para os frutos do tomateiro, em detrimento do crescimento das partes vegetativas (MARCELIS, 1993; BERTIN, 1993; DE KONNING, 1996). Plantas apresentando um maior número de folhas produzem mais assimilados, possuindo, portanto, um maior potencial de acumulação de matéria seca, que diminui a competição entre as partes vegetativas e os frutos. Essa situação foi observada na primavera, quando a radiação e a temperatura não foram limitantes (Tabela 1 e Figura 3a,c). Nesse experimento, a matéria seca total foi similar em todas as plantas até aproximadamente 90 dias da semeadura. Após esse período, as plantas com duas hastes mostraram uma tendência a um crescimento simultâneo da matéria seca vegetativa e de frutos, e a fração da matéria seca alocada para os frutos não foi reduzida em comparação com as plantas conduzidas com apenas uma haste (Tabela 2 e Figura 4a). Esse comportamento pode ser explicado pela hipótese citada, pois a elevada carga de frutos nas plantas com uma haste pode ter inibido o crescimento vegetativo, reduzindo a área foliar e a acumulação de matéria seca. Entretanto, esse comportamento não foi observado no outono. Nesse experimento, a fração de matéria seca nos frutos foi menor nas plantas conduzidas com duas hastes (Figura 4b). COCKSHULL et al. (1992) demonstraram que a acumulação de matéria seca de uma cultura de tomateiro depende basicamente da quantidade de energia solar interceptada pela cultura. Embora a radiação solar tenha sido mais baixa no outono, a acumulação de matéria seca total das plantas prosseguiu durante esse período, indicando que o crescimento dos frutos não foi inibido por uma limitação em nível da produção de assimilados pelas partes vegetativas. Entretanto, a produção extra de assimilados das plantas com duas hastes, decorrente de uma maior área foliar sob uma carga de frutos semelhante às plantas com uma haste, não foi alocada para os frutos, mas permaneceu como matéria seca vegetativa. Os resultados sugerem que a limitação ocorrida no crescimento dos frutos se deve ao efeito da temperatura do ar e que o transporte é pouco afetado pela temperatura; conclui-se que o efeito desse elemento meteorológico se exerce, provavelmente, sobre a força de dreno dos frutos. Em publicação anterior, HEUVELINK (1995) concluiu que a temperatura na faixa entre 18 e $24^{\circ} \mathrm{C}$ não afeta a repartição da matéria seca entre as partes vegetativas e os frutos do tomateiro. Porém, os resultados atuais mostraram que quando as temperaturas do ar apresentam fortes variações que ultrapassam aqueles limites, o crescimento dos frutos pode ser severamente afetado.

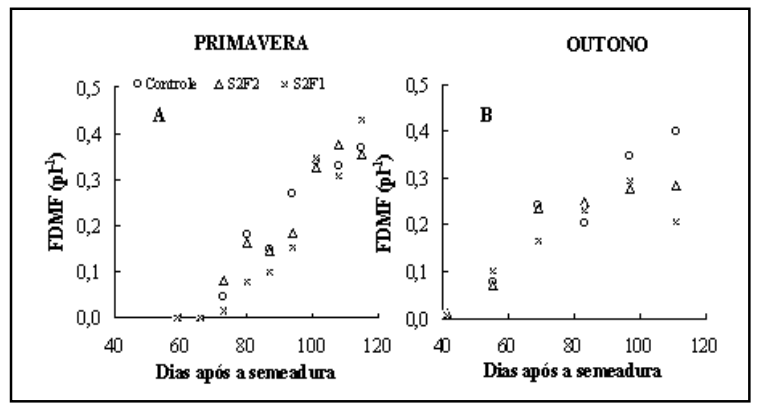

Figura 4 - Fração da matéria seca alocada para os frutos (FDMF) na primavera (a) e no outono (b). Santa Maria, RS, 1996 e 1997. 
Os resultados apresentados têm importantes consequiências para a produção comercial do tomateiro. Uma prática de manejo sugerida por COCKSHULL et al. (1992) foi de modificar o sistema de condução e de poda das plantas nos períodos de baixa radiação solar, a fim de aumentar a superfície de interceptação da luz e a produção de assimilados. Porém, essa prática só trará resultados positivos se as temperaturas do ar forem controladas. Por outro lado, um aumento na produção de matéria seca vegetativa não significa necessariamente um aumento na produção de frutos. Nesse caso, podas mais drásticas associadas com novas densidades de plantas devem ser testadas a fim de aumentar a fração da matéria seca alocada para os frutos.

\section{CONCLUSÕES}

A hipótese da existência de um "pool" único de assimilados circulando no interior da planta do tomateiro foi confirmada nas condições locais. A posição dos frutos sobre o caule não modificam a repartição da matéria seca entre as partes vegetativas e os frutos. No manejo das culturas, pode-se considerar a parte aérea das plantas como um único compartimento funcional.

\section{REFERÊNCIAS BIBLIOGRÁFICAS}

BERTIN, N. Environnement climatique, compétition pour les assimilats et modélisation de la nouaison de la tomate en culture sous serre. Paris, 1993. 120p. Thèse (Doctorat) Institut National Agronomique Paris-Grignon, 1993

BONNEMAIN, J.L. Transport $\mathrm{du}{ }^{14} \mathrm{C}$ assimilé chez les Solanacées. Revue Générale de Botanique, Paris, v.75, p.579-610, 1968.

BURIOL, G., STRECK, N.A., PETRY, C., et al. Transmissividade a radiação solar do polietileno de baixa densidade utilizado em estufas. Ciência Rural, Santa Maria, v.25, p.1-4, 1995.

CHALLA, H. Report of the working party 'crop growth models' Acta Horticulturae, Wageningen, v.174, p.169-175, 1985.

COCKSHUlL, K.E., GRAVES, C.J., CAVE, C.R.J. The influence of shading on yield of glasshouse tomatoes Journal of Horticulture Science, Ashford Kent, v.67, p.1124, 1992.

DE KONNING, A.N.M. Development and dry matter distribution in glasshouse tomato: a quantitative approach
Wageningen, 1994. 240p. Dissertation - Wageningen Agricultural University, 1994

ESTEFANEL, V., SCHNEIDER, F.M., BERLATO, $\boldsymbol{e}$ t al. Insolação e radiação solar na região de Santa Maria, RS: I Estimativa da radiação solar global incidente a partir dos dados de insolação. Ciência Rural, Santa Maria, v.20, p.203218,1990 .

FAO. Protected cultivation in the mediterranean climate Rome : Food and Agriculture Organization of the United Nations, 1990. 313p. FAO Plant Production and Protection Paper, 90.

HEUVELINK, E., MARCELIS, L.F.M. Dry matter distribution in tomato and cucumber. Acta Horticulturae, Wageningen, v.260, p.149-157, 1989.

HEUVELINK, E. Dry matter partitioning in a tomato plant: one common assimilate pool? Journal of Experimental Botany, Oxford, v.46, p.1025-1033, 1995.

HEUVELINK, E. Tomato growth and yield: quantitative analysis and synthesis. Wageningen, 1996. 326p. Dissertation - Wageningen Agricultural University, 1996.

HO, L.C. Metabolism and compartmentation of imported sugars in sink organs in relation to sink strength. Annual Review of Plant Physiology, Palo Alto, v.39, p.355-378, 1988.

HO, L.C., HEWITT, J.D. Fruit development. In: ATHERTON, J.G., RUDISCH, J. The tomato crop: a scientific basis for improvement. London : Chapman and Hall, 1986. p.201239

JONES, J.W., DAYAN, E., ALLEN, L.H. et al. A dynamic tomato growth and yield model (TOMGRO). Transactions of the American Society of Agricultural Engineers, Saint Joseph, v.34, p.663-672, 1991.

MARCELIS, L.F.M., BAAN HOFMAN-EIJER, L.R. Effect of temperature on the growth of individual cucumber fruits. Physiologia Plantarum, Copenhagen, v.87, p.321-328, 1993.

MARCELIS, L.F.M., DE KONNING, A.N.M. Biomass partitioning in plants. In: BAKER, J.C., BOT, G.P.A., CHALLA, H., et al. Greenhouse climate control. An integrated approach. Wageningen : Wageningen, 1995. p.84-92.

MARCELIS, L.F.M. Fruit growth and biomass allocation to the fruits in cucumber. 1. Effect of fruit load and temperature. Scientia Horticulturae, Amsterdam, v.54, p.107-121, 1993.

VEDUIN, J., BARTZ, H.R. Fertilidade do solo e rendimento do tomateiro em estufa de plástico. Ciência Rural, Santa Maria, v.28, n.2, p.229-233. 1998 .

WARREN-WILSON, J. 1972. Control of crop processes. In: REES, A.R., COCHSHULL, K.E., HAND, D.W., et al. Crop processes in controled environment. London : Academic, 1972. p.7-30. 